\title{
COMPACT DERIVATIONS OF NEST ALGEBRAS
}

\author{
C. PELIGRAD
}

\begin{abstract}
In this paper we determine all the weakly compact derivations of a nest algebra. We also obtain necessary and sufficient conditions in order that a nest algebra admit compact derivations. Finally we prove that every compact derivation of a nest algebra $\mathscr{A}$ is the norm limit of finite-rank derivations.
\end{abstract}

1. Let $\mathscr{A}$ be a Banach algebra and let $X$ be a Banach $\mathscr{A}$-module. By an $X$-valued derivation of $\mathscr{A}$ we mean a linear mapping $\delta: \mathscr{A} \rightarrow \mathscr{X}$ with the property $\delta(a b)=$ $a \delta(b)+\delta(a) b$ for all $a \in \mathscr{A}, b \in \mathscr{B}$. The derivation $\delta$ is called compact if $\delta$ is a compact operator between the Banach space $\mathscr{A}$ and $\mathscr{X}$, and weakly compact if $\delta$ is a weakly compact operator from $\mathscr{A}$ to $\mathscr{X}$ (i.e. $\delta\left(\mathscr{A}_{1}\right)$ is relatively weakly compact in $X$, where $\mathscr{A}_{1}$ is the unit ball of $\mathscr{A}$ [4]). Let $H$ be a complex Hilbert space, $B(H)$ the algebra of all bounded operators on $H$ and $\mathscr{K}(H)=\mathscr{K}$ the set of compact operators on $H$. In [7] Johnson and Parrott investigated derivations of a von Neumann subalgebra of $B(H)$ with range contained in $\mathscr{K}$. They proved that in most cases such derivations are implemented by a compact operator. The general result was recently obtained by Popa [8] who proved that this is the case for all von Neumann subalgebras of $B(H)$. Such derivations are known to be weakly compact [1].

On the other hand, in a series of papers $[1,9,10]$, C. A. Akemann, S. K. Tsui, and $\mathrm{S}$. Wright have determined the structure of all compact and weakly compact $\mathscr{A}$-valued derivations of a $C^{*}$-algebra $\mathscr{A}$, and of all compact $B(H)$ valued derivations of a $C^{*}$-subalgebra of $B(H)$.

In this note we determine the structure of all $\mathscr{A}$-valued compact and weakly compact derivations of a nest algebra $\mathscr{A}$. In particular we prove that every compact derivation of a nest algebra $\mathscr{A}$ is the norm limit of finite rank derivations. We need the following result.

LEMMA 1 [6]. Let $H$ be an infinite dimensional Hilbert space. If $\delta$ is a compact derivation of $B(H)$ then $\delta \equiv 0$.

Received by the editors January 16, 1984 and, in revised form, July 1, 1985.

1980 Mathematics Subject Classification. Primary 47D25; Secondary 47B05.

Key' words and phrases. Nest algebra, weakly compact derivation, compact derivation.

' Partially supported by NSF Grant No. DMS-8502353. 
2. In this section we state and prove our results on nest algebras.

Let $\mathscr{N} \cap \mathscr{B}(H)$ be a complete nest of projections, i.e. a totally ordered set of orthogonal projections which is strongly closed. We denote by $\mathscr{A}$ the nest algebra

$$
\operatorname{alg} \mathscr{N}=\{a \in \mathscr{B}(H) \mid p a p=a p, p \in \mathscr{N}\} \text {. }
$$

If $\mathscr{K} \cap B(H)$ is the set of compact operators on $H$ let $\mathscr{K}(\mathscr{A})=\mathscr{A} \cap \mathscr{K}$. It is known [5] that $\mathscr{K}(\mathscr{A})$ is ultraweakly dense in $\mathscr{A}$. A simple consequence of this and the duality between compact operators, trace class operators and $B(H)$ is the following.

LemMa 2. $\mathscr{K}(\mathscr{A})^{* *}=\mathscr{A}\left(\right.$ where $\mathscr{K}(\mathscr{A})^{* *}$ denotes the bidual of $\left.\mathscr{K}(\mathscr{A})\right)$.

We state now the analogue of [1, Theorem 3.1] for derivations of nest algebras.

THEOREM 3. Let $\delta$ be a derivation of $\mathscr{A}$. The following conditions are equivalent:

(1) $\delta$ is weakly compact.

(2) The range of $\delta$ is contained in $\mathscr{K}(\mathscr{A})$.

(3) $\delta=\operatorname{ad}(k)$ with $k \in \mathscr{X}(\mathscr{A})$.

Proof. (1) $\Rightarrow(2)$ By [2] $\delta$ is inner, and then $\delta(\mathscr{X}(\mathscr{A})) \subset \mathscr{K}(\mathscr{A})$. By Lemma 2 , $\mathscr{X}(\mathscr{A})^{* *}=\mathscr{A}$ whence $\delta=\left(\left.\delta\right|_{\mathscr{H}(\mathscr{A})}\right)^{* *}$. Since $\delta$ is weakly compact, $\left.\delta\right|_{\mathscr{X}(\mathscr{A})}$ is too, so by [4, Theorem VI.4.2] it follows that $\delta(\mathscr{A}) \subset \mathscr{K}(\mathscr{A})$.

(2) $\Rightarrow$ (3) follows immediately from [3, Theorem 1.4 and 2].

(3) $\Rightarrow$ (1) follows from the same implication of [1, Theorem 3.3].

The preceding result gives necessary and sufficient conditions for a derivation of a nest algebra to be weakly compact. Further, (Theorem 8 ) we give necessary and sufficient conditions for a nest algebra to admit a nonzero compact derivation.

We need the following well-known (and easy to prove) result.

Lemma 4. Let $\mathscr{A}$ be a nest algebra and let $\mathscr{N}=$ Lat $\mathscr{A}$. If $p \in \mathscr{N}$ then

$$
p B(X)(1-p) \subset \mathscr{A} \text {. }
$$

LemMA 5. If $\Lambda\{p \mid p \in \mathscr{N}, p \neq 0\}=0$ then $\mathscr{A}$ does not admit nonzero compact derivations.

Proof. The condition of the lemma implies $\operatorname{dim} p H=\infty$ for all $p \in \mathscr{N}, p \neq 0$. Let $\delta$ be a compact derivation of $\delta$. By Theorem $3, \delta=\operatorname{ad}(k)$ for some $k \in \mathscr{X}(\mathscr{A})$. We consider the following two possibilities:

(a) There is $p_{0} \in \mathcal{N}$, and $\xi_{0} \in\left(1-p_{0}\right) H,\left\|\xi_{0}\right\|=1$ such that $\left\langle k \xi_{0}, \xi_{0}\right\rangle \neq 0$ (here $\langle$,$\rangle denotes the inner product in H$ ). Let $k \xi_{0}=\lambda \xi_{0}+\eta_{0}$, where $\left\langle\xi_{0}, \eta_{0}\right\rangle$ $=0$ and $\lambda \neq 0$. Since $\operatorname{dim} p_{0} H=\infty$, let $\left\{\zeta_{n}\right\}_{n=1}^{\infty}$ be an orthonormal family of that space. We consider the operators $u_{n}:\left(1-p_{0}\right) H \rightarrow p_{0} H$ defined by

$$
u_{n} \xi_{0}=\xi_{n}, \quad u_{n}\left[\xi_{0}\right]^{\perp}=0, \quad n=1,2, \ldots
$$

By Lemma $4, u_{n} \in \mathscr{A}, n=1,2, \ldots$. Since $\left\|u_{n}\right\|=1$ for all $n$ and $\delta$ is compact, the sequence $\left\{\delta\left(u_{n}\right)\right\}$ contains a (norm) convergent subsequence which will be denoted by $\left\{\delta\left(u_{n}\right)\right\}$ too. On the other hand,

$$
\delta\left(u_{n}\right) \xi_{0}=k u_{n} \xi_{0}-u_{n} k \xi_{0}=k \zeta_{n}-\lambda \zeta_{n} .
$$


Since $k$ is compact and $\lambda \neq 0$, this sequence does not contain any convergent subsequence. This contradiction shows that in this case $k$ and so $\delta$ must be equal to zero.

(b) $\langle k \xi, \xi\rangle=0$ for all $\xi \in(1-p) H$ and all $p \in \mathscr{N}, p \neq 0$. In this case $(1-p) k=0$ for all $p \in \mathscr{N}$ and since $\wedge\{p \in \mathscr{N} \mid p \neq 0\}=0$, it follows that $k=0$ so $\delta=0$.

We denote $\mathscr{A}^{*}=\left\{a^{*} \in B(H) \mid a \in \mathscr{A}\right\}$. Then $\mathscr{A}^{*}$ is a nest algebra, and Lat $\mathscr{A}^{*}=\mathscr{N}^{c}=\{1-p \mid p \in N\}$.

Lemma $6 \cdot \operatorname{ad}(k), k \in \mathscr{K}(\mathscr{A})$, is a compact derivation of $\mathscr{A}$ if and only if $\operatorname{ad}\left(k^{*}\right)$ is a compact derivation of $\mathscr{A}^{*}$.

Proof. The proof is obvious.

Lemma 7. If $\wedge\{1-p \mid p \in \mathscr{N}, p \neq 1\}=0$ then $\mathscr{A}$ does not admit nonzero compact derivations.

Proof. The proof follows from Lemma 5 applied to $\mathscr{A}^{*}$, and Lemma 6.

Lemma 8. Let $\delta=\operatorname{ad}(k), k \in \mathscr{K}(\mathscr{A})$, be a compact derivation of a nest algebra $\mathscr{A}$. Then we have

(a) If $q_{0} \in \mathscr{N}(=$ Lat $\mathscr{A})$ is such that $\operatorname{dim}\left(1-q_{0}\right) H=\infty$ then $k q_{0}=0$.

(b) If $p_{0} \in \mathscr{N}$ is such that $\operatorname{dim} p_{0} H=\infty$, then $\left(1-p_{0}\right) k=0$.

Proof. (1) Suppose $k q_{0} \neq 0$. Then there is $\xi_{0} \in q_{0} H$ with $\left\|\xi_{0}\right\|=1, \zeta_{0} \neq 0$, such that $k \xi_{0}=\zeta_{0}$. Since $\operatorname{dim}\left(1-q_{0}\right) H=\infty$, there is an infinite orthonormal family $\left\{\zeta_{n}\right\}_{n=1}^{\infty}$ of that space. Consider the following family $\left\{u_{n}\right\}$ of operators, $u_{n}$ : $\left(1-q_{0}\right) H \rightarrow q_{0} H$ :

$$
u_{n} \zeta_{n}=\xi_{0}, \quad u_{n}\left[\zeta_{n}\right]^{\perp}=0, \quad n=1,2, \ldots
$$

Obviously, $\left\|u_{n}\right\|=1, n=1,2, \ldots$ By Lemma $4 u_{n} \in \mathscr{A}$ for all $n$. Since $\operatorname{ad}(k)$ is compact, the sequence $\left\{k u_{n}-u_{n} k\right\}_{n=1}^{\infty}$ contains a (norm) convergent subsequence which will be denoted by $\left\{k u_{n}-u_{n} k\right\}_{n=1}^{\infty}$ too. Therefore

$$
\lim _{m \geqslant n \rightarrow \infty}\left\|k\left(u_{n}-u_{m}\right)-\left(u_{n}-u_{m}\right) k\right\|=0 .
$$

In particular

$$
\lim _{m \geqslant n \rightarrow \infty}\left\|k\left(u_{n}-u_{m}\right) \zeta_{n}-\left(u_{n}-u_{m}\right) k \zeta_{n}\right\|=0 .
$$

Since $k$ is compact and $\left\{\zeta_{n}\right\}$ is an orthonormal family, we have $\lim _{n \rightarrow \infty}\left\|k \zeta_{n}\right\|=0$. Since $\left\|u_{n}\right\|=1$ for all $n$ we also have $\lim _{m \geqslant n \rightarrow \infty}\left\|\left(u_{n}-u_{m}\right) k \zeta_{n}\right\|=0$, so

$$
\lim _{m \geqslant n \rightarrow \infty}\left\|k\left(u_{n}-u_{m}\right) \zeta_{n}\right\|=0 \text {. }
$$

But $k\left(u_{n}-u_{m}\right) \zeta_{n}=k u_{n} \zeta_{n}=k \xi_{0}=\zeta_{0} \neq 0$. This contradiction proves the lemma. To prove (2) we apply the preceding argument to $\mathscr{A}^{*}$ and $1-p_{0}$ (instead of $\mathscr{A}$ and $\left.q_{0}\right)$. We now state our necessary and sufficient conditions for $\mathscr{A}$ to admit a nonzero compact derivation. 
THEOREM 9. Let $\mathscr{A}$ be a nest algebra and $\mathscr{N}=$ Lat $\mathscr{A}$. The following are equivalent:

(1) $\mathscr{A}$ admits a nonzero compact derivation.

(2) There is a $p \in \mathscr{N}, p \neq 0$, with $\operatorname{dim} p H<\infty$ and a $q \in \mathscr{N}, q \neq 1$, such that $\operatorname{dim}(1-q) H<\infty$.

Proof. (2) $\Rightarrow$ (1) Indeed let $k \in p B(H)(1-q) \subset \mathscr{A}$. Then, it is easy to see that $\operatorname{ad}(k)$ is a compact (actually a finite rank) derivation of $\mathscr{A}$.

$(1) \Rightarrow(2)$ Suppose first that $\operatorname{dim} p H=\infty$ for all $p \in \mathscr{N}, p \neq 0$. Let $p_{0}=$ $\wedge\{p \in \mathscr{N} \mid p \neq 0\}$. Then, either $p_{0}=0$ or $\operatorname{dim} p_{0} H=\infty$. If $p_{0}=0$ then by Lemma 5, $\mathscr{A}$ does not admit nonzero compact derivations. Assume $p_{0} \neq 0$ and let $\delta$ be a compact derivation of $\mathscr{A}$. By Theorem $3, \delta=\operatorname{ad}(k)$ for some $k \in \mathscr{K}(\mathscr{A})$. Since $\mathscr{A}$ is a nest algebra, it follows that $p_{0} \mathscr{A} p_{0}=B\left(p_{0} H\right)$. Since $k p_{0}$ implements a compact derivation of $p_{0} \mathscr{A} p_{0}=B\left(p_{0} H\right)$ and $\operatorname{dim} p_{0} H=\infty$, by Lemma 1 we have $k p_{0}=p_{0} k p_{0}=0$. On the other hand, by Lemma 8 , we have $\left(1-p_{0}\right) k=0$. Therefore $k=p_{0} k\left(1-p_{0}\right)$. It remains to prove that $p_{0} k\left(1-p_{0}\right)=0$. Assume the contrary. Then there is $\zeta \in\left(1-p_{0}\right) H, \zeta \neq 0$, with $k \zeta=\xi_{0} \in p_{0} H,\left\|\xi_{0}\right\|=1$. Since $\operatorname{dim} p_{0} H=\infty$, let $\left\{\zeta_{n}\right\}_{n=1}^{\infty}$ be an orthonormal family of that infinite dimensional space. Let $\left\{u_{n}\right\}_{n=1}^{\infty} \subset B\left(p_{0} H\right)=p_{0} \mathscr{A} p_{0} \subset \mathscr{A},\left\|u_{n}\right\|=1$ be the family of operators defined by

$$
u_{n} \xi_{0}=\zeta_{n}, \quad u_{n}\left[\xi_{0}\right]^{\perp}=0, \quad n=1,2, \ldots
$$

Then, since $\operatorname{ad}(k)$ is a compact derivation of $\mathscr{A}$, the sequence $\left\{\left(k u_{n}-u_{n} k\right) \zeta\right\}_{n=1}^{\infty}$ contains a norm convergent subsequence. But $\left(k u_{n}-u_{n} k\right) \zeta=-u_{n} k \zeta=-\zeta_{n}$ so this sequence does not contain any convergent subsequence. This contradiction shows that $p_{0} k\left(1-p_{0}\right)=0$ and hence $k=0$. Assume now that $\operatorname{dim}(1-q) H=\infty$ for all $q \in \mathscr{N}, q \neq 1$. Then, the preceding argument applied to $\mathscr{A}^{*}$ shows that $\mathscr{A}^{*}$ does not admit nonzero compact derivations. By Lemma $6, \mathscr{A}$ does not admit nonzero compact derivations and the proof of Theorem 9 is complete.

THEOREM 10. Every compact derivation of a nest algebra $\mathscr{A}$ is the norm limit of finite rank derivations.

Proof. Let

$$
p_{0}=\bigvee\{p \in \mathscr{N} \mid \operatorname{dim} p H<\infty\} \quad \text { and } \quad q_{0}=\bigwedge\{q \in \mathscr{N} \mid \operatorname{dim}(1-q) H<\infty\}
$$

Let also $\delta=\operatorname{ad}(k), k \in \mathscr{K}(\mathscr{A})$, be a compact derivation of $\mathscr{A}$. We first prove that $k=p_{0} k\left(1-q_{0}\right)$. We note that $\left(1-p_{0}\right) k$ implements a compact derivation of the nest algebra $\left(1-p_{0}\right) \mathscr{A}\left(1-p_{0}\right)$. If $\operatorname{dim} p_{0}<\infty$ then $\operatorname{Lat}\left(1-p_{0}\right) \mathscr{A}\left(1-p_{0}\right)$ does not contain any finite dimensional projection, so by Theorem $9,\left(1-p_{0}\right) k=0$. If $\operatorname{dim} p_{0}=\infty$ we have $\left(1-p_{0}\right) k=0$ by Lemma $8(\mathrm{~b})$. Hence $k=p_{0} k$. A similar argument shows that $k=k\left(1-q_{0}\right)$. From the definition of $p_{0}$ (respectively $q_{0}$ ) it follows that there exists a sequence $\left\{p_{n}\right\} \subset \mathscr{N}, \operatorname{dim} p_{n} H<\infty$ (respectively $\left\{q_{n}\right\} \subset$ $\mathcal{N}, \operatorname{dim}\left(1-q_{n}\right) H<\infty$ ) such that $p_{n} \uparrow p_{0}$ (respectively $q_{n} \downarrow q_{0}$ ); of course, if $\operatorname{dim} p_{0} H<\infty$ (respectively $\left.\operatorname{dim}\left(1-q_{0}\right) H<\infty\right)$, the sequence $\left\{p_{n}\right\}$ (respectively 
$\left.\left\{q_{n}\right\}\right)$ will be constant. Since $k$ is compact, it follows that

$$
k=p_{0} k\left(1-q_{0}\right)=(\text { norm }) \lim _{n \rightarrow \infty} p_{n} k\left(1-q_{n}\right) .
$$

As obviously ad $\left(p_{n} k\left(1-q_{n}\right)\right)$ is finite rank the proof of Theorem 10 is complete.

\section{REFERENCES}

1. Ch. Akemann and S. Wright, Compact and weakly compact derivations of $C^{*}$-algebras, Pacific J. Math. 85 (1979), 253-259.

2. E. Christensen, Derivations of nest algebras, Math. Ann. 229 (1977), 155-161.

3. E. Christensen and C. Peligrad, Commutants of nest algebras module the compact operators, Invent. Math. 56 (1980), 113-116.

4. N. Dunford and J. T. Schwartz, Linear operators, Part I, Interscience, New York, 1958.

5. J. A. Erdos, Operators of finite rank in nest algebras, J. London Math. Soc. 43 (1968), 391-397.

6. Y. Ho, A note on derivations, Bull. Inst. Math. Acad. Sinica 5 (1977).

7. B. E. Johnson and S. K. Parrott, Operators commuting with a von Neumann algebra modulo the set of compact operators, J. Funct. Anal. 11 (1972), 39-61.

8. S. Popa. The commutant modulo the set of compact operators of a von Neumann algebra, preprint, Increst, 1985.

9. S. K. Tsui and S. Wright, Asymptotic commutants and zeroes of von Neumann algebras, Math. Scand. 51 (1982), 232-240.

10. S. Wright, Banach-module-valued derivations on $C^{*}$-algebras, Illinois J. Math. 24 (1980), 462-467.

Department of Mathematical Sciences, University of Cincinnati, Cincinnati, Ohio 45221 